\title{
Development of a Finite Element Digital Human Hand Model
}

\author{
Gregor HARIH*1, Mitsunori TADA ${ }^{2}$ \\ ${ }^{1}$ Laboratory for Intelligent CAD systems, Faculty for Mechanical Engineering, \\ University of Maribor, Maribor, Slovenia; \\ ${ }^{2}$ Digital Human Research Group, Human Informatics Research Institute, \\ National Institute of Advanced Industrial Science and Technology, Japan
}

DOI: $10.15221 / 16.208 \quad$ http://dx.doi.org/10.15221/16.208

\begin{abstract}
The aim of this research was to develop an anatomically accurate and numerically feasible and stable finite element digital human hand model, which would allow accurate bio-mechanical behavior during movement and grasping. Therefore, correct anatomical geometry of the hand has been defined based on CT images, which has been then used for the definition of the finite element model. Using the finite element software, material properties and boundary conditions have been defined to obtain accurate movement of the bones and deformation of the skin. The result of the conducted research is a developed angle-driven finite element digital human hand model, which is numerically stable. Using the accurate geometry and correct definition of the material properties and boundary conditions, the finite element digital human hand model shows reasonable bio-mechanical behavior under movement.
\end{abstract}

Keywords: digital human model, finite element method, simulation, human hand, bio-mechanics

\section{Introduction}

The human hand is one of the most sophisticated bio-mechanical tools of a human. The main function of the hand is the interaction with the physical environment, where the most important is the prehensile hand grasp with a physical object. Thereby it can be effectively used as a tool for work, as well as an interface to use various powered and non-powered hand tools and products. Product designers need to consider due to the nature of certain complex tasks the appropriate product-human interaction to develop products with high rate of performance, comfort and safety. Modern Computer Aided Engineering (CAE) and Computer Aided Design (CAD) software allow the designer to design and evaluate new products virtually [1]. Product designers have to consider ergonomics to increase the human-product performance, comfort, and lower the risk of cumulative trauma disorders (CTD) [2]. CTDs can be defined as a set of syndromes, which can be characterized as discomfort with persistent pain in the muscles, tendons, and other soft tissue; and joint movement inability or with reduced mobility. The most common CTDs as a result of usage of handheld products are bursitis, carpal tunnel syndrome, hand-arm vibration syndrome, ischemia, white finger syndrome, etc. [3]. It has been shown that CTDs result for major sick leaves of workers, which presents high costs for the company and high costs with diagnostics and treatment [4].

Traditional user-centered design techniques, such as designing with recommendations, designing based on anthropometric data and derived mathematical models and ergonomic analyses using prototypes, costly measurement systems and iterative design process increases the time and cost of the product development process [5]. Additionally, these methodologies do not incorporate enough data to design products with best fit for a target population. This extensive ergonomic knowledge that is necessary during the design phase of a handheld product; and its poor integration with existing, well-established CAD software, has affected companies that do not or on very low scales address these ergonomic principles resulting in poor ergonomics of the developed products [5].

Most researchers also focused on the sizes and the shapes of the handheld products, but neglected those materials of the product that are in direct contact with the user's hand. It has been shown that successful choice of product material is critical for success of the whole product on the market [6]. Authors provided basic guidelines for material choice, but did not investigate the mechanical behaviour of the skin and soft tissue whilst grasping product surfaces of different materials [7].

To overcome limitations of traditional design, there has been an increase in use of multidisciplinary methods to reverse engineer functional 3D CAD models of human anatomical parts, so called digital human models (DHM) to study bio-mechanics, incorporate them into the design process using CAD models or to utilize Finite Element Analyses (FEA) and therefore compute stresses on the biological tissue and propose design changes to increase the resulting comfort and safety of the product [8]. For example, 3D models of human foot based on medical imaging are increasingly being used in the design process of footwear to optimize the performance and comfort [9]. 
Usually those hand-arms of DHMs that are parts of a whole digital human models are used to evaluate vision, clearance and tasks [10]. However, these models do not incorporate the possibility to evaluate the design in terms of mechanical stresses and deformations on biological tissue, which have been shown to be major contributor to injury frequency. Therefore those hands of DHM that only consider the kinematics of the hand, but neglect the anatomical shape of the hand and soft tissue deformation whilst grasping, cannot be used for realistic bio-mechanical and ergonomic analyses [11]. The mechanical behaviour of the biological materials of human hand is crucial during the human-product interaction, since forces and moments are transferred from the product to anatomical structures [12]. The nature of the human hand and complex surfaces of the products usually prevent the direct measurements of stresses, strains and contact pressure, therefore it has been already shown that the only usable method in virtual environment are computer simulations using finite element (FE) method [13].

The purpose of the proposed interdisciplinary research was to develop a finite element digital human hand model (FE-DHHM), which would allow simultaneous studying of bio-mechanics of human hand movement and grasping, analyses of biological tissue deformations, internal stresses, contact pressures and also effects of vibration on the hand. In terms of product development, the FE-DHHM would allow direct evaluation of the proposed handheld product design inside virtual environment with topological modifications of the product to lower the peak stresses, deformation and contact pressures and also reduce the effects of vibration on the whole hand-arm system. This would also lower the designing time and cost of the products. Based on these analyses, safety and performance could be predicted and design errors could be identified and corrected during the design phase to increase performance, comfort and avoid CTD. In this paper we present the development of the FE-DHHM and first steps in movement simulations.

\section{Material and methods}

\subsection{Finite element model - material properties}

Fingertip bone and nail were assumed to be linear elastic with isotropic material parameters with Young's modulus of 17GPa and 170MPa respectively, with a Poisson ratio of 0.3 [12].

The behaviour of soft tissue can be described with hyper-elastic material models, where one of the most used is the Ogden strain energy potential [14] (equation 1):

$$
U=\sum_{i=1}^{N} \frac{2 \mu_{i}}{\alpha_{i}^{2}}\left[\bar{\lambda}_{1}^{\alpha_{i}}+\bar{\lambda}_{2}^{\alpha_{i}}+\bar{\lambda}_{3}^{\alpha_{i}}-3+\frac{1}{D_{i}}\left(J^{e l}-1\right)^{2 i}\right]
$$

where $\bar{\lambda}_{i}$ are the deviatoric principal stretches; $\bar{\lambda}_{i}=J^{-\frac{1}{3}} \lambda_{i} ; \lambda_{i}$ are the principal stretches; $N$ is number of terms used in strain energy function; and $\mu_{i}, \alpha_{i}$ are temperature-dependent material parameters and describe shear behaviour of the material, $D_{i}$ is compressibility, $J$ is the Jacobean determinant and $J_{e l}$ is the elastic volume ratio. The initial shear modulus and bulk modulus for the Ogden form are given in equation 2.

$$
\mu_{0}=\sum_{i=1}^{N} \mu_{i}, \quad K_{0}=\frac{2}{D_{1}}
$$

Compressibility can be defined with the specified non-zero values for $D_{i}$, where the Poisson ratio is less than 0.5 and is expressed in equation 3.

$$
D_{i}=\frac{2}{K_{0}}=\frac{3(1-2 v)}{\mu_{0}(1+v)}
$$

The material parameters for soft tissue were extracted from a uniaxial tensile test, and were fitted to the Ogden hyper-elastic material model and are provided in Table 1 with used equations for the numerical calculations [15]. As soft tissue is almost incompressible, the Poisson ratio was determined to be 0.45 [16]. We considered quasi-static simulations with low deformation rates, therefore the viscosity of the soft tissue was unconsidered in our simulations. Same material properties have been used by us in our previous research and showed great correspondence to experiments [13, 17].

Table 1: Material parameters determining hyper elasticity of soft tissue:

\begin{tabular}{|c|c|c|}
\hline $\mathrm{N}$ & $\mu_{i}$ & $\boldsymbol{\alpha}_{\mathrm{i}}$ \\
\hline 1 & -0.07594 & 4.941 \\
\hline 2 & 0.01138 & 6.425 \\
\hline 3 & 0.06572 & 4.712 \\
\hline
\end{tabular}




\subsection{Geometry acquisition based on medical imaging}

Due to the nature of the human body, most appropriate method in tissue three-dimensional data acquisition is Magnetic Resonance Imaging (MRI) and Computed Tomography (CT), which has been already used by us in the past for the development of a static DHHM [18, 19]. Therefore, for the development of the proposed FE-DHHM CT medical imaging has been undertaken. The optimal posture for the generation of the FE model was determined to obtain undeformed soft tissue of the hand. Obtained images were manually segmented into bones (phalanxes, metacarpal bones, carpal bones, radius and ulna) and skin in medical imaging software ITK-SNAP [20]. Obtained models of the anatomical structures were then exported in STL file format and water-tight IGES models have been generated in Geomagic software. Afterwards all anatomical structures were imported into the Abaqus FE software for the appropriate definition of the FE model (figure 1).

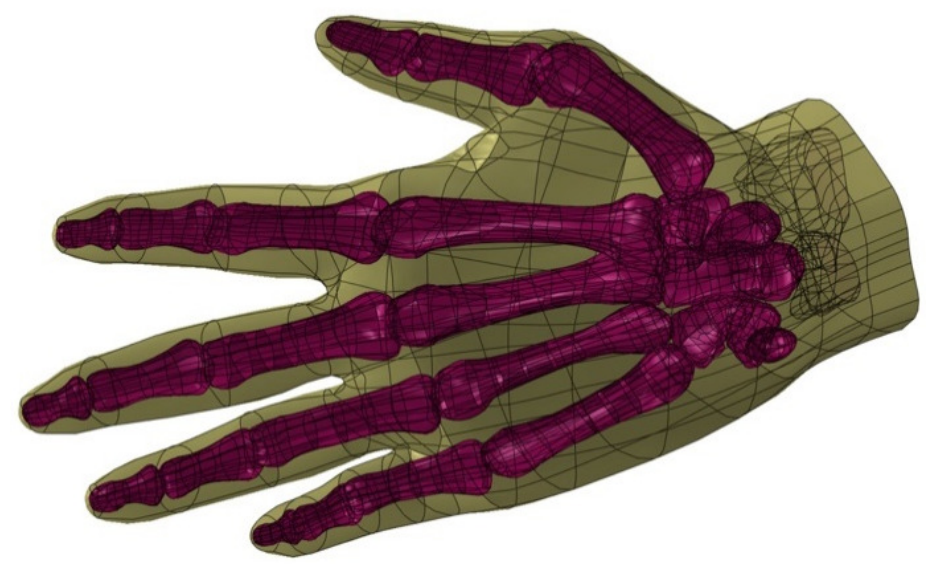

Fig. 1. Geometry definition based on medical images.

\subsection{Finite element model - geometrical and boundary conditions}

The bio-mechanical movement of human hand is determined based on geometry and material properties of the human hand, where bone link structure with ligaments and tendons is one of the most important [21]. Our definition of a joint between distal and proximal phalange is shown in Fig. 2. Firstly, we identified the center of the rotation in proximal phalange bone where a new local coordinate system was created. The coordinate system was oriented in such manner that the " $y$ " axis corresponded with the axis of the rotation of the joint axis. In the center of the new local coordinate system a new reference point (RP-PIP) was created. Another reference point (RP-DIP) was also created on the surface of the distal phalange bone. Both reference points were connected to bones using constraints, which fixed their translations and rotations relative to the bone. Between both reference points a rigid wire has been created, which has been then used to define a connector that allows rotation only in " $y$ " axis at the RP-PIP point. This way a simplified, numerically stable and bio-mechanically correct joint can be defined.

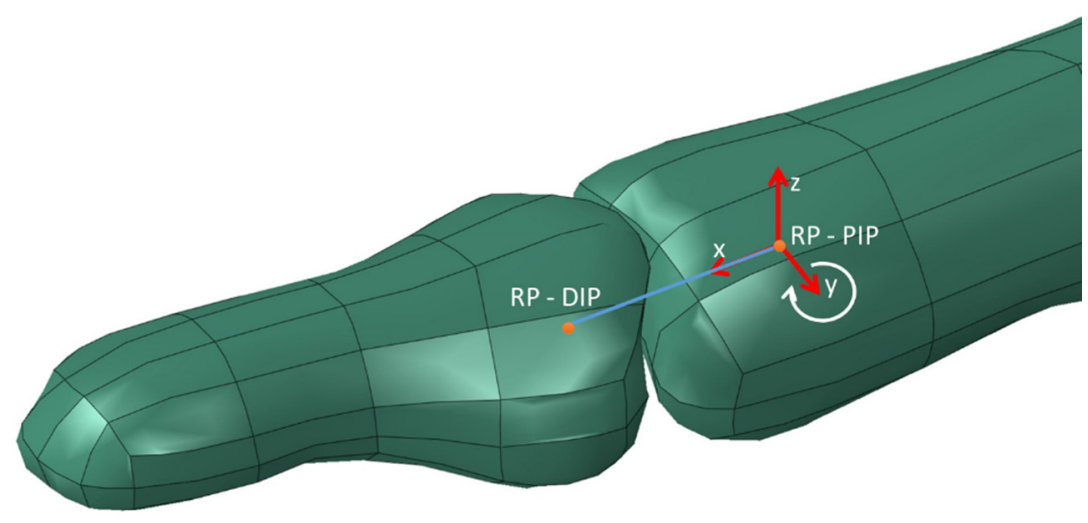

Fig. 2. Definition of a joint.

Other joints in human hand have been defined analogously considering their local features. Fully defined FE-DHHM consisted of 30 constraints and 15 wires/connectors. Based on all definitions of the joints 18 DOF of the FE-DHHM have been created (Fig. 3). 


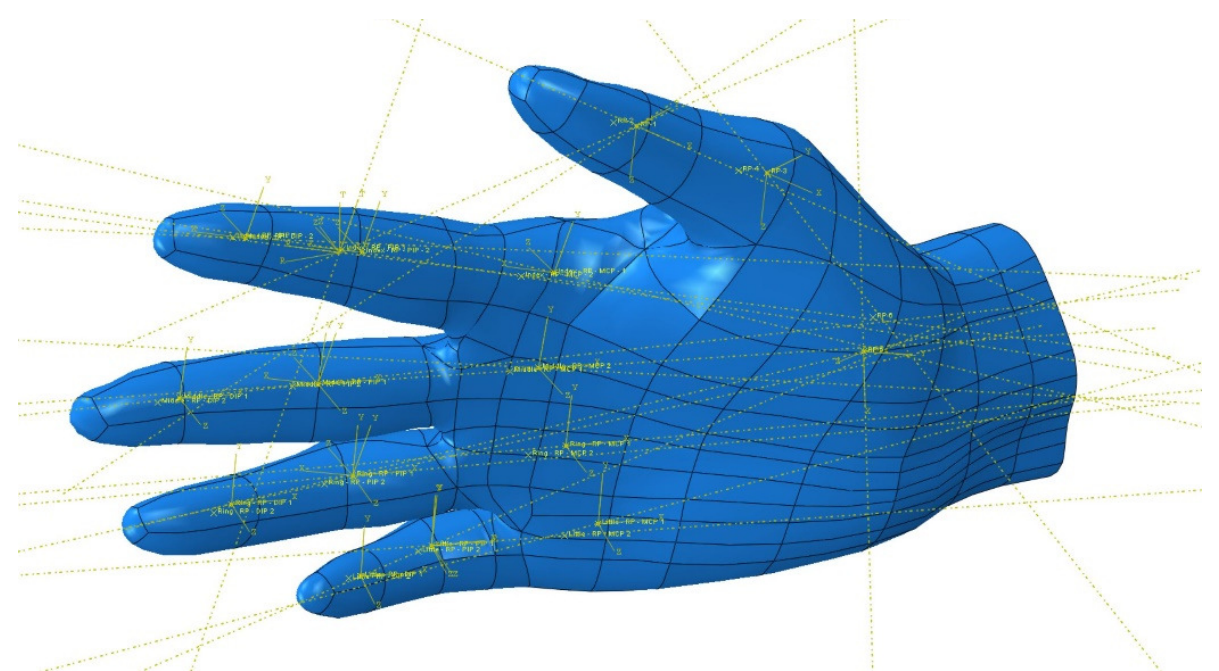

Fig. 3. Fully defined FE hand model.

In order to be able to perform numerical tests, we created a mesh using 326427 4-node linear tetrahedral elements (C3D4) as shown in Fig. 4.

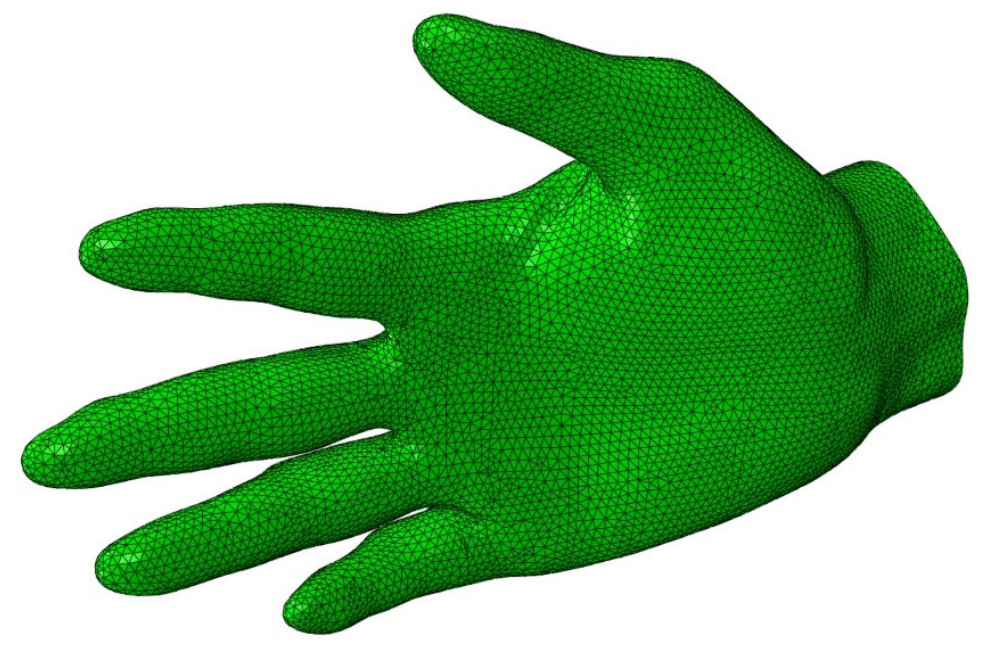

Fig. 4. Meshed FE hand.

\subsection{Finite element model - numerical tests}

Based on the joint link structure our FE-DHHM is joint angle driven. Therefore, the joint movement is simulated using the connectors connecting two adjacent bones into one joint. The rotation of the joint is specified in the manner of degree of rotation. In our initial numerical test each joint has been prescribed with 1 radian $\left(57,3^{\circ}\right)$ of rotation. In this manner rotations at each joints could be observed under same boundary conditions.

\section{Results and discussion}

FE-DHHM have not been developed yet due complexity of the human hand anatomy and resulting bio-mechanics. We have shown that a successful development of such bio-mechanical system requires simplifications, which is a compromise between accuracy of the bio-mechanical behavior of the model and model complexity and calculation times. Since the presented FE-DHHM is one of the first full hand finite element models we introduced several simplifications in terms of anatomical structures, material models and joint definitions. Based on our previous research, we have shown that such simplifications are reasonable and allow maintaining high level of accuracy of the simulated system.

Despite described simplifications, the developed FE-DHHM firstly showed convergence problems, which could be addressed to mesh irregularities. They occurred due to complex geometry of the hand anatomy. The problem with convergence was solved using various geometry repair tools and locally 
finer mesh. Afterwards the FE-DHHM proved to be stable since the final step of numerical calculation has been achieved. The final calculation time for the prescribed boundary conditions was 47 minutes using two cores on an Intel Xeon (E5-2695V2) processor. The final obtained posture of the FE-DHHM can be observed in Fig. 5.

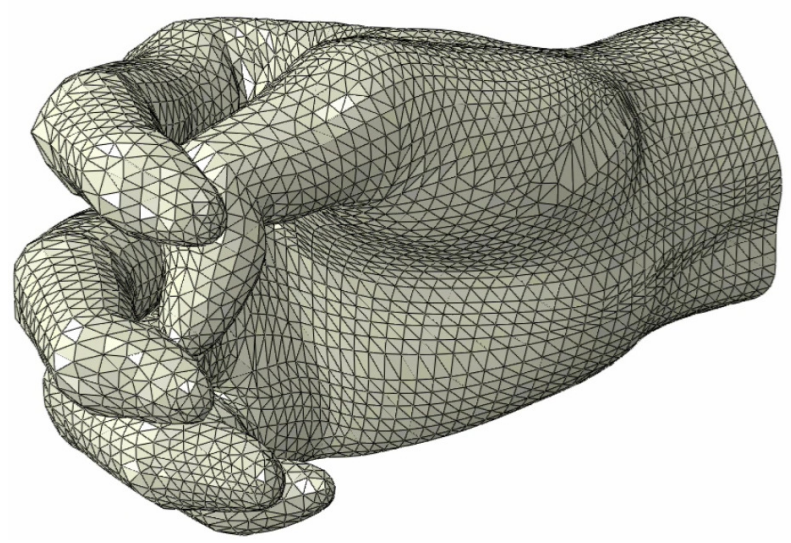

Fig 5. Final posture of the FE-DHHM after numerical calculations.

The soft tissue deformation of the FE-DHHM during simulation is a consequence of the bone link structure movement prescribed by the boundary conditions. Therefore, we carefully investigated the bone movement, which is defined with the joint definitions. We observed that joint definitions were defined correctly since each bone showed correct bio-mechanical movement (Fig. 6.).
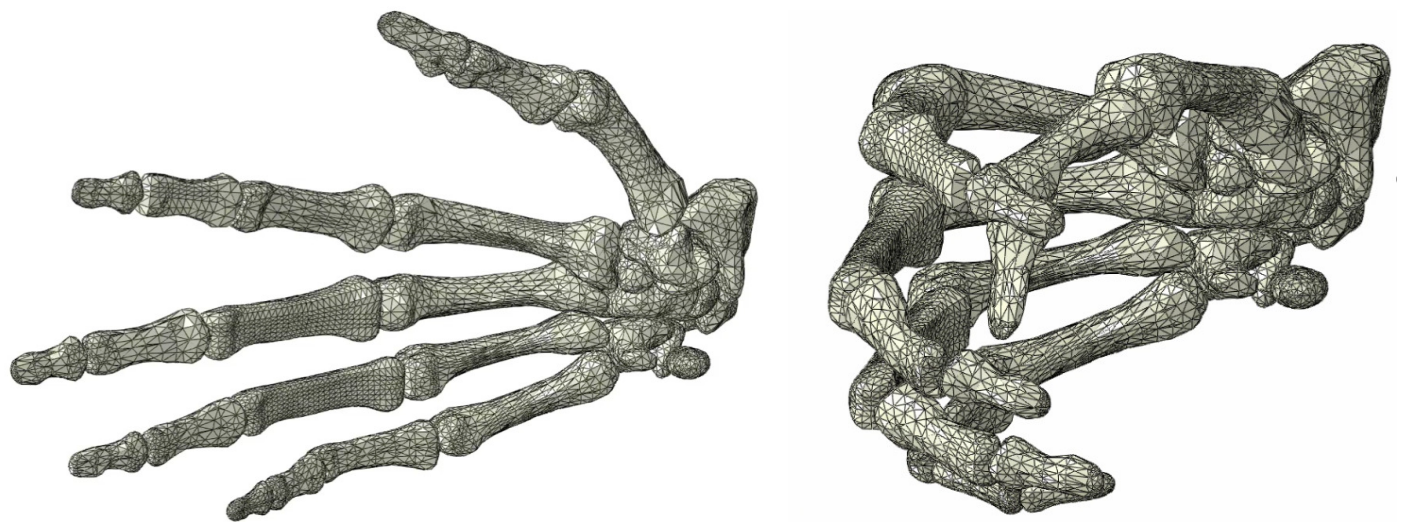

Fig. 6. Bone structure responsible for the deformation of soft tissue.

The simplified joint introduced in this paper is numerically low-cost and provides accurate movement of the bones. Therefore, the movement and deformation of soft tissue can be also considered as bio-mechanically accurate, since the material model has been verified and validated by us in previous papers. The simplified joint model using the connectors on the other hand cannot be used for obtaining and assessing results in terms of loads on the joints (forces, moments, contact pressures).

Real-world grasping is very complex and is also depends on the subjective comfort rating of the user, therefore this kind of evaluation can be unreliable. Therefore, the FE-DHHM can be also easily coupled to an appropriate motion capture system where joint angles of hand movements can be extracted and feed into our FE model from real life grasping scenarios. Hereby realistic hand posture and grasping pattern can be obtained, which can provide results in terms of soft tissue stresses, strains and also contact pressures when an object is grasped. Several researchers have shown that a power grasp produces a very uneven distribution of contact pressure on the hand and fingers, which can lead to discomfort, pain, and acute and also cumulative traumatic disorders. Using the FE-DHHM these areas can be identified and design changes can be undertaken to avoid such issues.

In this regards future work should include throughout process of verification and validation of the developed FE-DHHM. Future FE-DHHM should also include more anatomical structures (skin, subcutaneous tissue, nail, capsules, synovial fluid...) and more realistic joint definitions to obtain more realistic numerical results. Additionally, the FE-DHHM should be updated to allow grasping of various objects and studying ergonomic and also bio-mechanic aspects of new product development ultimately leading to injury identification and also prevention. 


\section{Conclusion}

We have shown that deformations and stresses that represent basic results of the structural analysis using FE method are also an important aspect in the field of ergonomics. In this manner finite element method has been successfully used to develop an angle driven full hand finite element human hand model. Initial simulations have shown that the FE-DHHM is numerically feasible and also stable, which presents a reasonable bio-mechanical movement during simulations.

\section{References}

[1] M. Bordegoni and C. Rizzi, Innovation in Product Design: From CAD to Virtual Prototyping: Springer, 2011.

[2] G. Salvendy, Handbook of Human Factors and Ergonomics: John Wiley \& Sons, 2012.

[3] A. Moore, R. Wells, and D. Ranney, "Quantifying exposure in occupational manual tasks with cumulative trauma disorder potential," Ergonomics, vol. 34, pp. 1433-1453, Dec 1991.

[4] S. Tanaka, D. K. Wild, L. L. Cameron, and E. Freund, "Association of occupational and non-occupational risk factors with the prevalence of self-reported carpal tunnel syndrome in a national survey of the working population," American Journal of Industrial Medicine, vol. 32, pp. 550-6, 1997.

[5] J. Kaljun and B. Dolšak, "Ergonomic design knowledge built in the intelligent decision support system," International Journal of Industrial Ergonomics, vol. 42, pp. 162-171, 2012.

[6] L. Y. Ljungberg and K. L. Edwards, "Design, materials selection and marketing of successful products," Materials \& Design, vol. 24, pp. 519-529, 2003.

[7] G. L. Fellows and A. Freivalds, "Ergonomics evaluation of a foam rubber grip for tool handles," Applied Ergonomics, vol. 22, pp. 225-30, 1991.

[8] H. O. Demirel and V. G. Duffy, "Applications of Digital Human Modeling in Industry," Lecture notes in computer science, pp. 824-832, 2007.

[9] P. Franciosa, S. Gerbino, A. Lanzotti, S. Patalano, V. Rufrano, M. Trotta, et al., "Virtual prototype development for comfort assessment of occupational footwear sole," in Proceedings of the IMProVe 2011 International conference on Innovative Methods in Product Design, 2011, pp. 487-494.

[10] D. B. Chaffin and G. Andersson, Occupational biomechanics. New York: Wiley, 1999.

[11] O. A. van Nierop, A. van der Helm, K. J. Overbeeke, and T. J. P. Djajadiningrat, "A natural human hand model," The Visual Computer: International Journal of Computer Graphics, vol. 24, pp. 31-44, 2007.

[12] J. Z. Wu and R. G. Dong, "Analysis of the contact interactions between fingertips and objects with different surface curvatures," Proceedings of the Institution of Mechanical Engineers, Part H: Journal of Engineering in Medicine, vol. 219, pp. 89-103, 2005.

[13] G. Harih and B. Dolšak, "Recommendations for tool-handle material choice based on finite element analysis," Applied Ergonomics, vol. 45, pp. 577-585, 2014.

[14] A. Ali, M. Hosseini, and B. Sahari, "A review of constitutive models for rubber-like materials," American Journal of Engineering and Applied Sciences, vol. 3, pp. 232-239, 2010.

[15] L. Pan, L. Zan, and F. S. Foster, "Ultrasonic and viscoelastic properties of skin under transverse mechanical stress in vitro," Ultrasound in Medicine and Biology, vol. 24, pp. 995-1007, Sep 1998.

[16] J. Z. Wu, R. G. Dong, S. Rakheja, and A. W. Schopper, "Simulation of mechanical responses of fingertip to dynamic loading," Medical Engineering \& Physics, vol. 24, pp. 253-264, May 2002.

[17] G. Harih and M. Tada, "Finite element evaluation of the effect of fingertip geometry on contact pressure during flat contact," International Journal for Numerical Methods in Biomedical Engineering, vol. 31, pp. n/a-n/a, 2015.

[18] G. Harih and B. Dolšak, "Tool-handle design based on a digital human hand model," International Journal of Industrial Ergonomics, vol. 43, pp. 288-295, 2013.

[19] G. Harih and A. Čretnik, "Interdisciplinary Approach to Tool-Handle Design Based on Medical Imaging," BioMed Research International, vol. 2013, pp. 1-8, 2013.

[20] P. A. Yushkevich, J. Piven, H. C. Hazlett, R. G. Smith, S. Ho, J. C. Gee, et al., "User-guided 3D active contour segmentation of anatomical structures: Significantly improved efficiency and reliability," YNIMG Neurolmage, vol. 31, pp. 1116-1128, 2006.

[21] C. L. Taylor and R. J. Schwarz, "The anatomy and mechanics of the human hand," Artificial limbs, vol. 2, pp. 22-35, 1955. 